\title{
Facile and Green Synthesis of Multicolor Fluorescence Carbon Dots from Curcumin: In Vitro and in Vivo Bioimaging and Other Applications
}

\author{
Tathagata $\mathrm{Pal}^{\dagger, \S}$ Shanid Mohiyuddin, ${ }^{\ddagger, \S}$ and Gopinath Packirisamy ${ }^{*}, \dagger, \ddagger(0)$
}

${ }^{\dagger}$ Nanobiotechnology Laboratory, Centre for Nanotechnology and ${ }^{\ddagger}$ Department of Biotechnology, Indian Institute of Technology Roorkee, Roorkee, Uttarakhand 247667, India

\section{Supporting Information}

ABSTRACT: Early detection is the critical phase in the prognostic strategy of various life-threatening maladies like infectious diseases and cancer. The mortality rate caused by these diseases could be considerably reduced if they were diagnosed in the early stages of disease development. Carbon dots (C-dots), a relatively new and promising candidate in the fluorescent nanomaterial category, possess a perceptible impact on various bioapplications. Herein, we report a one-step facile hydrothermal synthesis that yields a novel surface-passivated carbon dot (CDP) from curcumin (as a green substrate) displaying high aqueous solubility. The physico-chemical characterization of thus synthesized C-dots was accomplished by an UV-visible spectrophotometer, fluorescence spectrophotometer, zetasizer, TEM, and FE-SEM to understand the formation of carbon dots with a 4-5 nm size near spherical nanoparticle with high colloidal stability. E. coli DH5 $\alpha$ was engaged as the Gram-negative test organism and S. aureus as the Gram-positive in the biolabeling of bacteria. Cancer cell lines including colon cancer (HCT-15), lung cancer (A549), and mouse fibroblast (NIH 3T3) were evaluated and resulted in good biolabeling potential and less cytotoxicity. Zebrafish (ASWT) embryos as an animal model system were bioimaged, and in vivo toxicity was inferred. Moreover, the synthesized C-dots were shown to have free radical scavenging activity in a dose-dependent manner. The unpassivated C-dots (CD) were found to sense ferric ions at the micromolar concentration level. The findings of our study suggest that the multifunctional potentiality of CDPs serves as high-performance optical nanoprobes and can be a suitable alternative for various biolabeling and contrasting agents.

\section{INTRODUCTION}

In recent days, nanotechnology has extended its nonavoiding potential influence in the fields of basic science to prototype application. Basically, nanotechnology deals with materials at the nanoscale level owing to vast tailoring possibilities, resulting in different composition size and shape of materials. The nanometer size enables them with unique properties which can further be modified according to required applications. ${ }^{1}$ Luminescent carbon dots (C-dots) are comparatively new additions to this field, showing enormous applications in health, energy, and environment. Although it shares similarities with quantum dots, it has the advantage of being nontoxic as compared to the heavy-metal-based quantum dots. After its discovery in 2006, it has gained the massive attention of research groups in the field of bioanalytics as well as bioimaging due to its efficient fluorescent property ${ }^{2}$ and high biocompatibility. $^{3}$

C-dots are basically carbon nanoparticles with size below 10 $\mathrm{nm}$. The prepared C-dots show the chemical composition of nitrogen, hydrogen, and oxygen and other basic elements with respect to the substrate of preparation. Synthesis of C-dots is made possible in multiple ways including arc discharge, laser ablation, electrochemical, hydrothermal, ultrasonic, microwave, and solvothermal processes. Hydrothermal synthesis is the best among them, forming high quantum yield C-dots with the advances in controlling the size, composition, purity, energy and cost-effectiveness. Furthermore, thus synthesized C-dots can evade the phase transition from mesophase to substable phase. The basis of fluorescence property in carbon dots is still unknown. Mechanistically, the excitation-dependent emission behaviours of $\mathrm{C}$-dots might be due to the various surface defects caused during the synthesis, leading to various energy traps. The activation of different surface energy traps results in different fluorescence properties. More interestingly, the fluorescence from the C-dots can be quenched efficiently by electron donor or acceptor molecules in the solution which can make it a good agent for metal ion sensing. C-dots can be passivated by various functional groups to cover the carbon core of the C-dots for increasing the quantum yield (QY), better chemical stability, and maximum aqueous solubility. C-

Received: September 7, 2017

Accepted: January 2, 2018

Published: January 23, 2018 


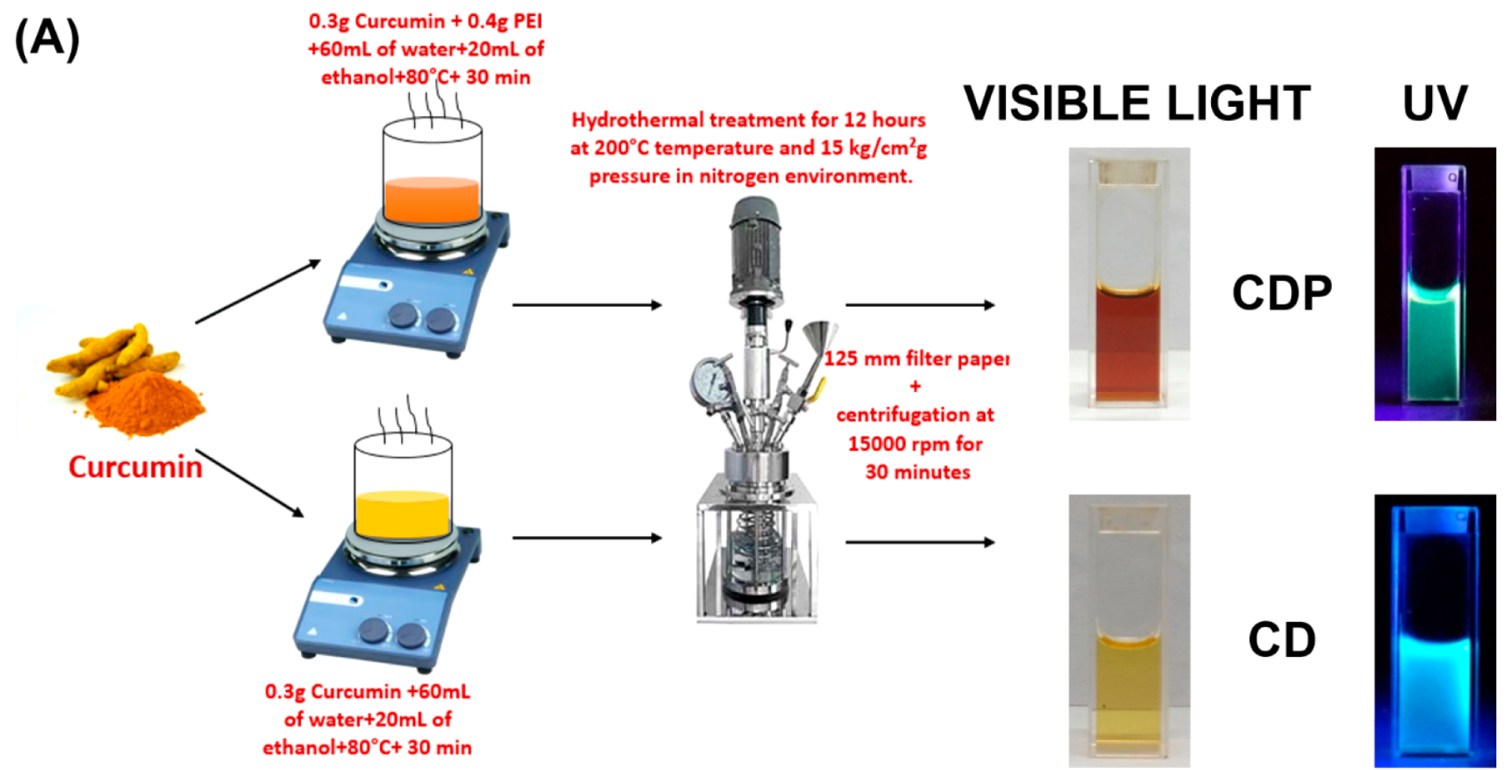

(B)

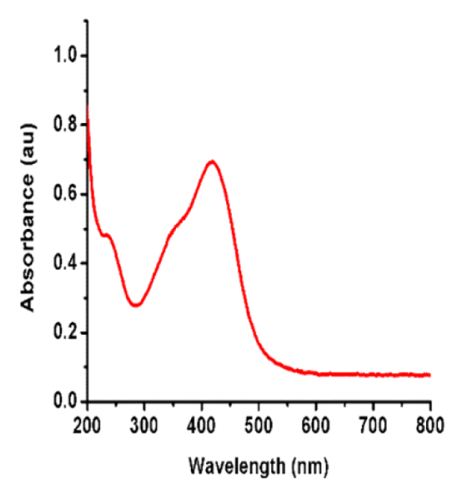

(C)

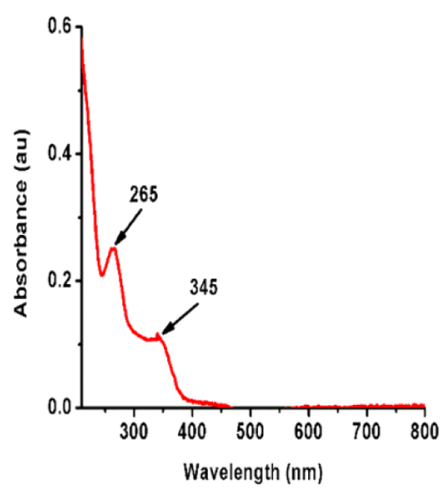

(D)

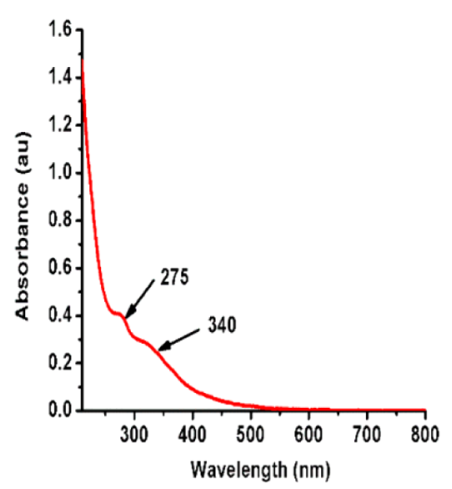

Figure 1. (A) Schematic representation of the synthesis of C-dots through the hydrothermal reactor followed by visualization of CDP and CD under visible light and UV light differentiates the UV excited fluorescence. UV-vis absorbance spectra of (B) curcumin alone, (C) CD, and (D) CDP.

dots with emission in the near-infrared (NIR) spectral region can also be used in biobased applications of nanobiotechnology. NIR emission of C-dots is very significant since the body tissues are transparent in the NIR water window.

The theranostic potential of $\mathrm{C}$-dots gives the opportunity for biolabeling, and thereby early diagnosis and management of diseases by loading therapeutic medicine ${ }^{4}$ enables a speedy recovery. C-dots possess better biocompatibility, in vivo stability, fewer side effects, low cost of production, and control of intellectual property rights for the successful theranostic implication. One similarity of most of the carbohydrate-based $\mathrm{C}$-dots is that the diffraction peak shows amorphous nature between $2 \theta=18-24{ }^{\circ} \mathrm{C}$. $^{5-7} \mathrm{C}$-dots stay in the amorphous stage with lattice spacing closely resembling graphitic and turbostratic carbon. To synthesize C-dots various carbon sources were reported like lamp soot, ${ }^{8}$ food caramels, ${ }^{9}$ soya milk, ${ }^{10}$ orange juice, $^{11}$ eggshell, ${ }^{12}$ protein (bovine serum albumin), ${ }^{13}$ and watermelon. ${ }^{14}$ However, scaling up the synthesis procedure is still a challenging task.

Curcumin is the principle compound found in Curcuma longa (commonly known as turmeric) giving a yellow color to the turmeric. It can exist in several tautomeric forms including a 1,3-diketo form and two equivalent enol forms. The enol form is more energetically stable in the solid form and in solution.
Curcumin has antioxidants, anticancerous, anti-inflammatory, antiviral, and antifungal activity along with metal sensing properties. However, poor aqueous solubility leading to very low bioavailability delimits its efficiency as a potential agent for application in diverse fields. There are no reports dealing with the solubility issue of the curcumin despite of its huge potentials in applications. However, the formation of carbon dot from the curcumin brings about enhanced aqueous solubility and can cross over the delimiting factor. The successful synthesis of highly green fluorescent carbon dots surface passivated with oligoethyleneamine (OEI) resulted in enhanced biocompatibility and can be used as potential nanoprobes for biomedical application. ${ }^{15}$ Herein, we are reporting for the first time the synthesis of carbon dots from curcumin and surface passivated with branched PEI (bPEI) giving rise to increased quantum yield (QY), better chemical stability, and enhanced aqueous soluble C-dots. These assynthesized C-dots were used as a potential biolabeling agent along with antioxidation and metal ion sensing properties.

\section{RESULTS AND DISCUSSION}

2.1. Characterization of Synthesized CDP and C-Dots. The color of CDP and CD was brown and pale yellow under visible light and ocean green and bright blue under UV light, 
(A)

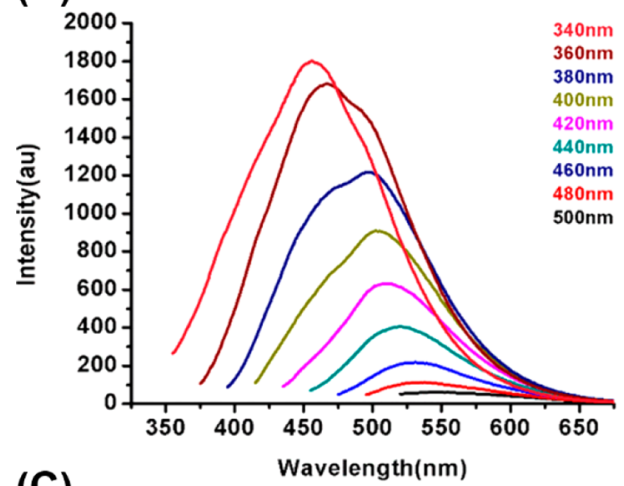

(C)

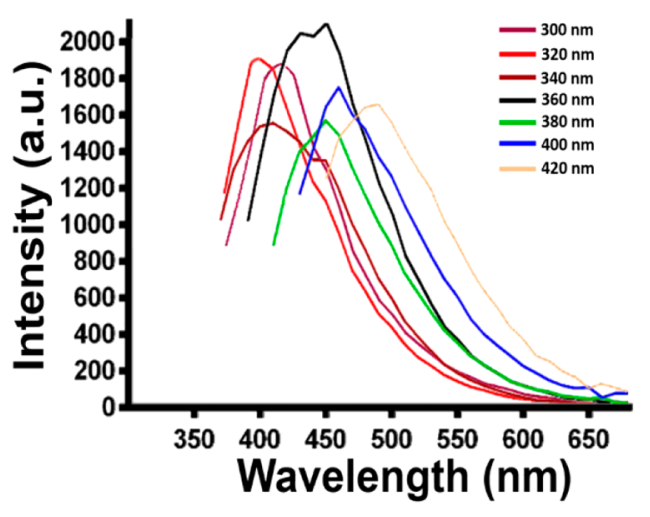

(B)

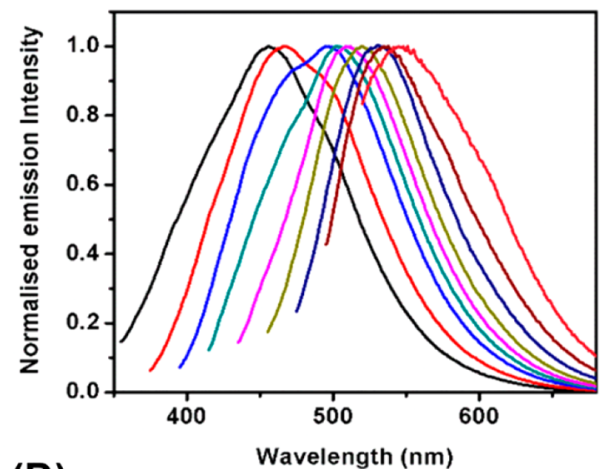

(D)

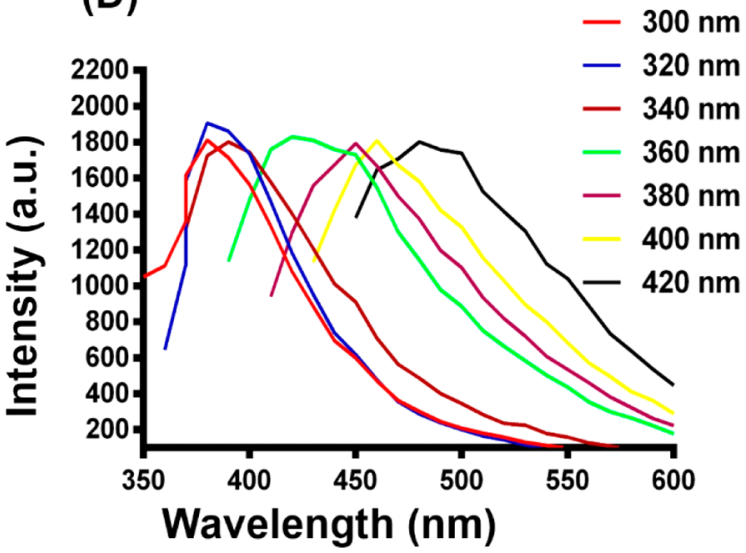

Figure 2. Excitation-dependent fluorescence emission of (A) CDP with excitation from 340 to $500 \mathrm{~nm}$ and (B) normalized excitation-dependent emission intensity of CDP. Emission spectra of (C) CD and (D) PEI alone hydrothermal treated carbon dots with respect to different excitation.

(A)

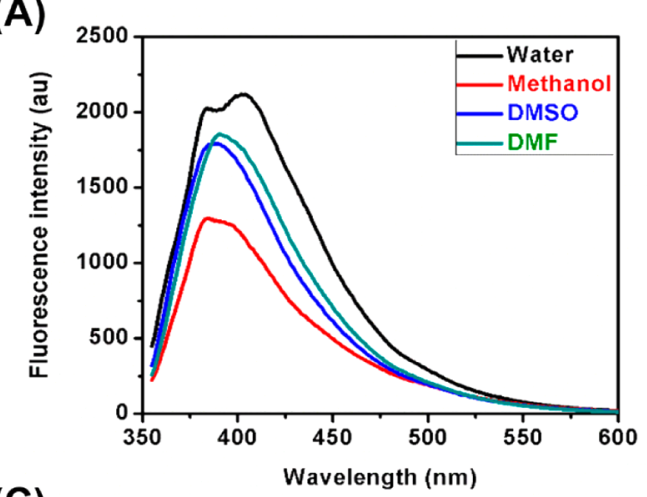

(C)

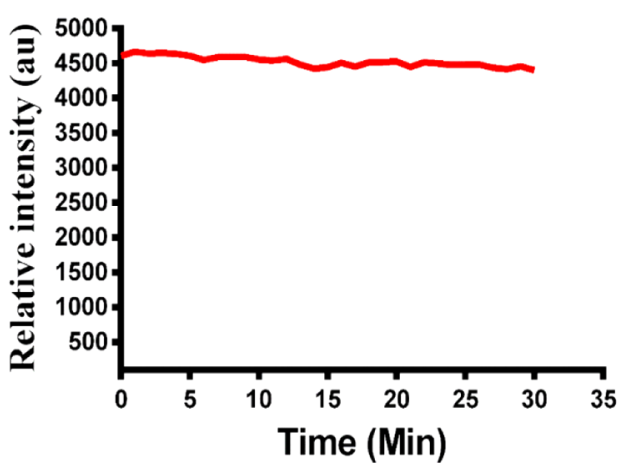

(B)

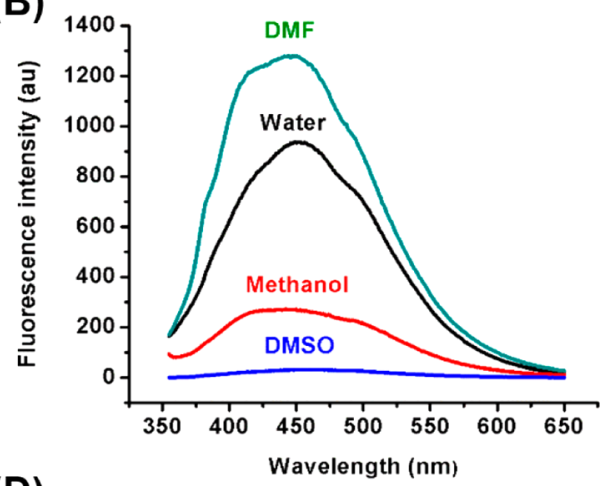

(D)

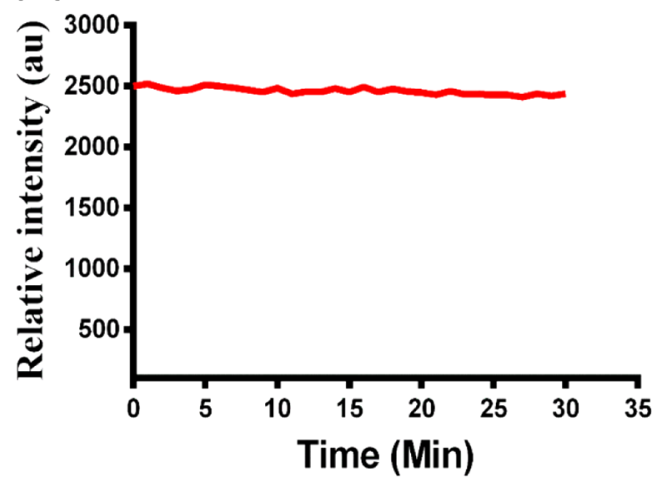

Figure 3. Effect of different solvents including water, methanol, DMSO, and DMF on fluorescence emission in (A) CD and (B) CDP. The photostability study of (C) CD and (D) CDP under constant excitation with a xenon lamp for $30 \mathrm{~min}$. 
(A)

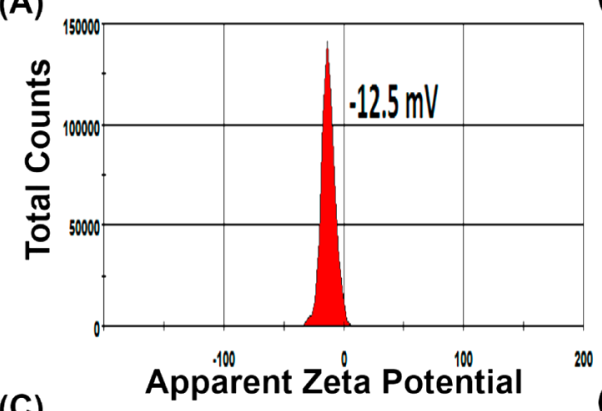

(C)

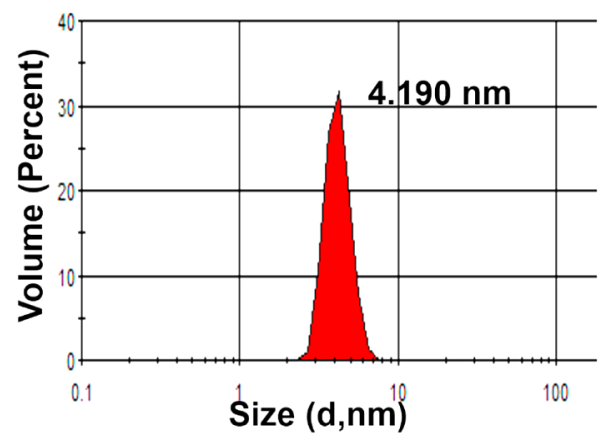

(B)

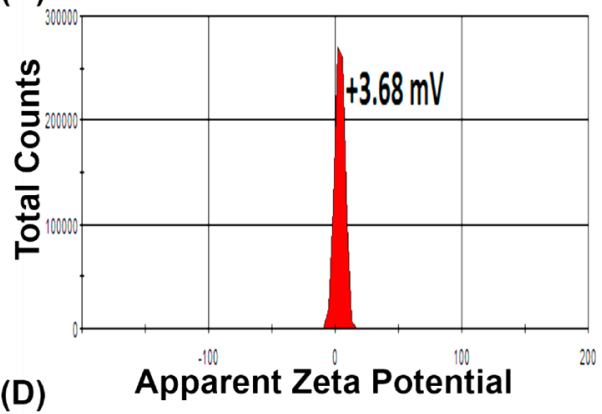

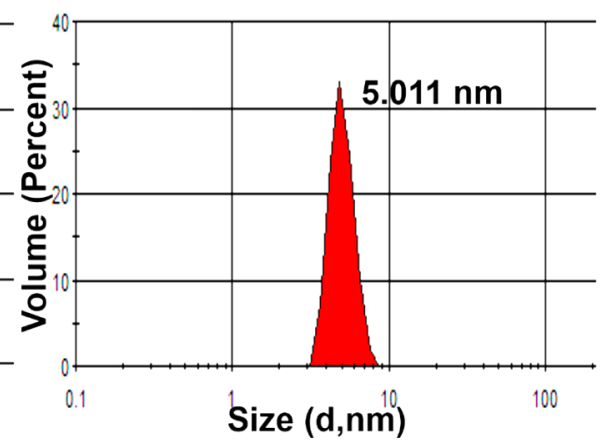

Figure 4. Zeta potential of (A) CD and (B) CDP. The hydrodynamic diameter of (C) CD and (D) CDP.

(A)

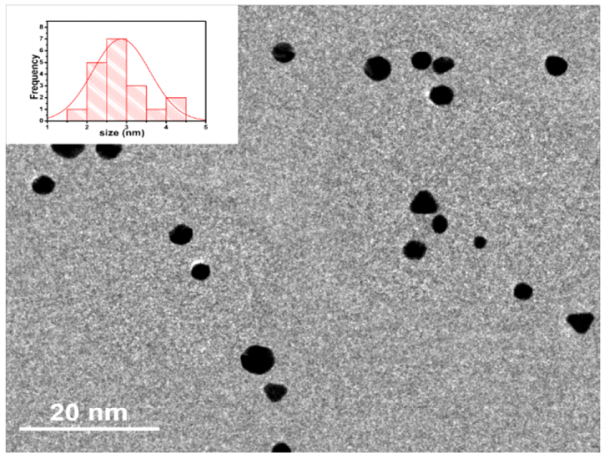

(B)

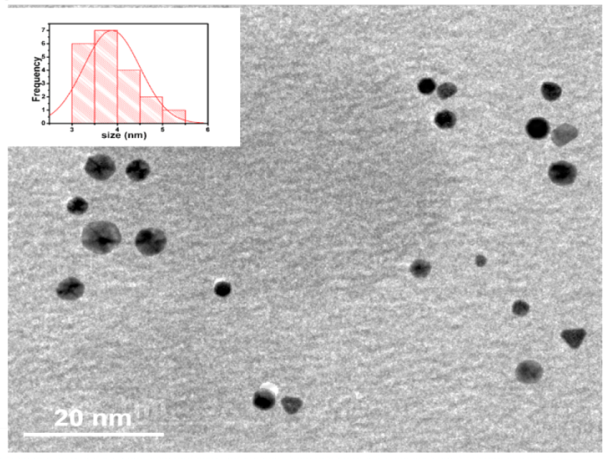

(C)

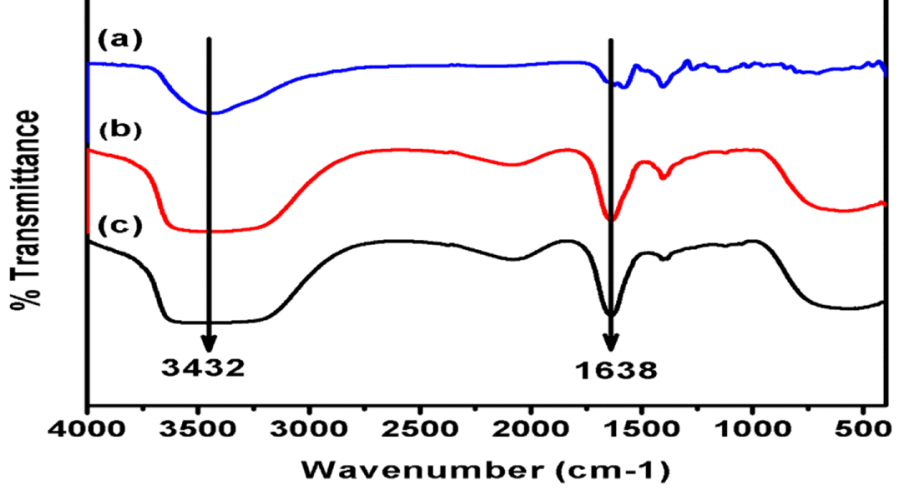

Figure 5. High-resolution transmission electron microscopic (HRTEM) image of (A) CD and (B) CDP with inset revealing the size distribution. (C) FTIR spectra of (blue line) curcumin, (red line) CD, and (black line) CDP.

respectively, as shown in Figure 1A. In UV-vis analysis the CD shows two absorption bands at $265 \mathrm{~nm}\left(\pi-\pi^{*}\right.$ transition $)$ and $345 \mathrm{~nm}\left(\mathrm{n}-\pi^{*}\right.$ transition) as in Figure 1C, whereas CDP portrays two absorption bands at $275 \mathrm{~nm}\left(\pi-\pi^{*}\right.$ transition $)$ and $340 \mathrm{~nm}\left(\mathrm{n}-\pi^{*}\right.$ transition) as demonstrated in Figure 1D. The absorbance of CDP increases from the 265 to $275 \mathrm{~nm}$ onset of the surface passivation. The fluorescence spectroscopic study revealed that the maximum fluorescence emission intensity of $\mathrm{CD}$ was due to excitation at $280 \mathrm{~nm}$, and for CDP it was $340 \mathrm{~nm}$ (Figure S1). Since PEI is a well-known precursor for the carbon dot synthesis as well as surface passivation agent resulting in high biocompatible excellent fluorescent carbon dots, ${ }^{16}$ we underwent comparative analysis of the fluorescence activity of carbon dots prepared from the 
(A)

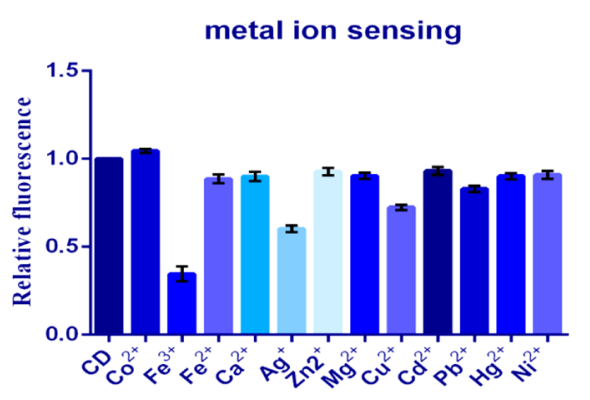

(C)

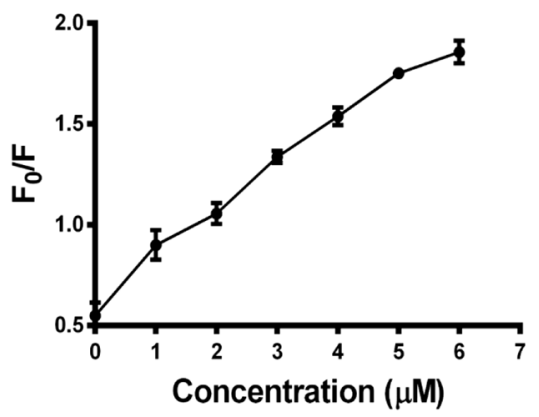

(B)
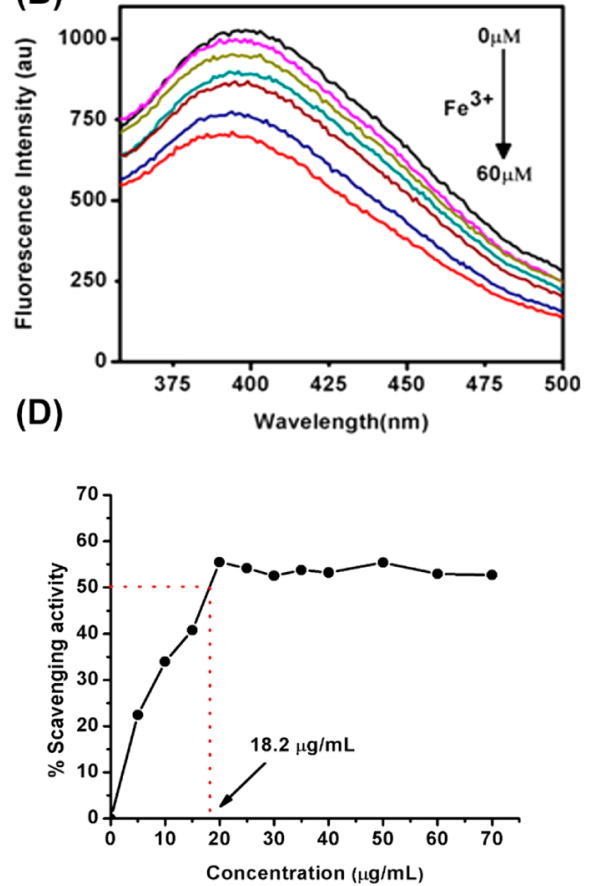

Figure 6. (A) Relative fluorescence emission intensity quenching by 12 metal ions. The values are represented as mean \pm SEM $(n=3)$. (B) Fluorescence quenching of $\mathrm{CD}$ with the addition of different concentrations of $\mathrm{Fe}^{3+}$. (C) Relative fluorescence response values $\left(F_{0} / F\right)$ of $\mathrm{CD}$ with respect to $0-6 \mu \mathrm{M}$ concentration of $\mathrm{Fe}^{3+}$ (D) DPPH free radical scavenging activity of CDP.

curcumin alone (CD), PEI alone, and PEI passivated curcumin (CDP) carbon dots. It was observed with the synergistic effect in the fluorescence behavior of CDP (Figure 2A) with respect to the same of $\mathrm{CD}$ (Figure 2C) and PEI alone (Figure 2D) carbon dots.

As-synthesized CD and CDP were further dissolved in water, methanol, dimethylformamide (DMF), and dimethyl sulfoxide (DMSO) to investigate the effect of the solvent on the fluorescent behavior. In both the carbon dots, it is found that the water dissolved sample is harvested with a promising fluorescent yield (Figure 3A and B). This clearly indicates the high solubility of synthesized C-dots toward universal solvent and does not hinder the fluorescent activity. Also, in CDP, maximum fluorescence was observed in DMF. This might be due to the presence of the amide group on the surface of the carbon dots which was further verified by FTIR. Furthermore, both carbon dots did not display any significant reductions in emission intensity onset of excitation by a $150 \mathrm{~W}$ Xe lamp for about $30 \mathrm{~min}$ as demonstrated in Figure 3C and Figure 3D. The improved photostability enriches the efficiency of CDP and C-dots as a potential biological labeling agent. Quantum yield was measured and represented as in Table S1 by a singlepoint method and found to be $2.28 \%$ and $8.607 \%$ for CDP and $\mathrm{CD}$, respectively. All the fluorescent-based characterizations showed positive results toward the efficiency of C-dots in susceptible usage in the biomedical scenario.

2.2. Zeta Potential and DLS (Hydrodynamic Diameter) Analysis. Curcumin owns a high negative zeta potential of -26 $\mathrm{mV} .{ }^{17}$ The zeta potential of CD was $-12 \mathrm{mV}$ as in Figure 4A. This is due to the destruction of some of the functional groups of curcumin upon the carbon dots synthesis. The positive charge bearing PEI resulting upon passivation made considerable changes in the zeta potential of the CDP and turned out to be $+3.68 \mathrm{mV}$ (Figure 4B). The amide bond was formed due to the reaction of the keto group of curcumin and the amine group of PEI. This resulted in less amino group being present on the surface of CDP, making the zeta potential less positive as a cumulative surface charge. The hydrodynamic size of the CD was found to be $4.190 \mathrm{~nm}$ (Figure 4C), and that of CDP was $5.011 \mathrm{~nm}$ (Figure. 4D). The increased size of the C-dots upon passivation ensures that the knowledge of efficient passivation with PEI was correct and corroborates the zeta potential results as mentioned above.

2.3. TEM and SAED Analysis. The size and nature of the phase of carbon dots was examined by high-resolution transmission electron microscopy (HRTEM). HRTEM images depict smaller, near spherical formation of nanoparticles. For $\mathrm{CD}$ the average diameter was found to be $3.28 \mathrm{~nm}$ with the perspective of Figure 5A, and for CDP it was $4.45 \mathrm{~nm}$ (Figure $5 B$ ). The mean size was obtained by using imageJ2 software analysis. Overtaking proper dilution of carbon dots, the hydrodynamic diameter was found to be $4.19 \mathrm{~nm}$ for CD and $5.011 \mathrm{~nm}$ for CDP by dynamic light-scattering assay. In both of the cases, the diameter is slightly higher than that measured in the TEM micrograph. The possible reason for this fluctuation is due to the aqueous environment present in the DLS assay, while dry samples were micrographed in HRTEM. Furthermore, SAED patterns of carbon dots showed diffused rings indicating the amorphous phase of the nanoparticles (Figure S2) in both CD and CDP. The amorphous nature of carbon dots ${ }^{18}$ is indicating the carbon dots were properly synthesized.

2.4. Fourier Transform Infrared (FTIR) Spectroscopic Analysis. The chemical characterization of CD and CDP were further extended toward the FTIR spectral assay. In the fingerprint region $\left(1300-400 \mathrm{~cm}^{-1}\right)$ a noticeable change is observed from carbon dots to curcumin alone. The smoothening of the curves in Figure 5C (red and black lines) 
(A)

UV-2A (330-380 nm) $(450-490 \mathrm{~nm})$

G-2A $(510-560 \mathrm{~nm})$
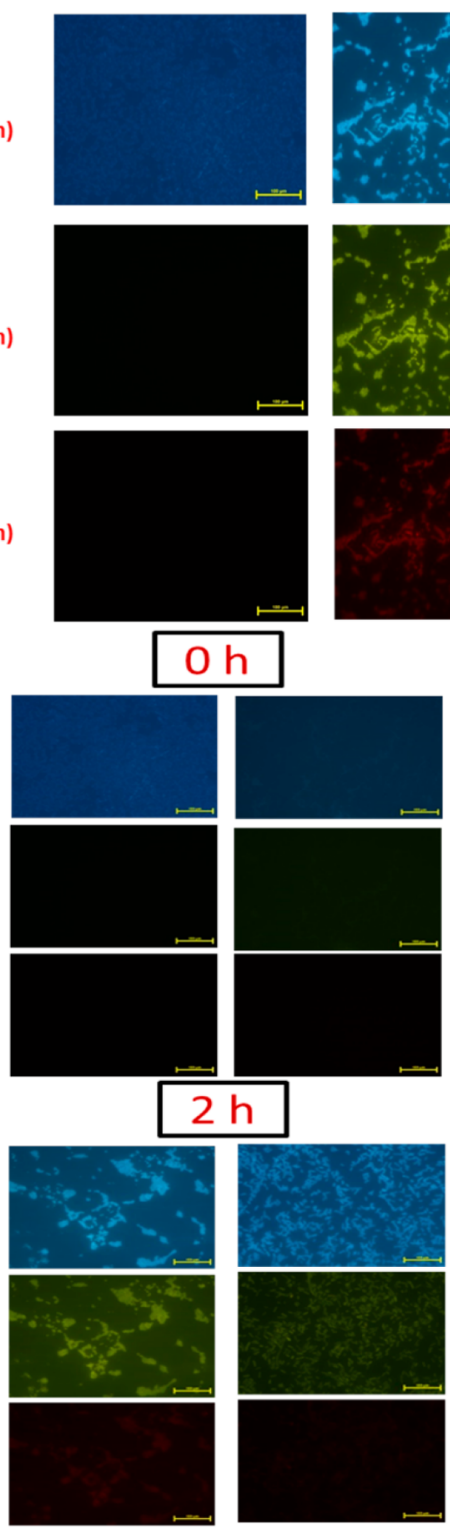

(B)

S. aureus + CDPs
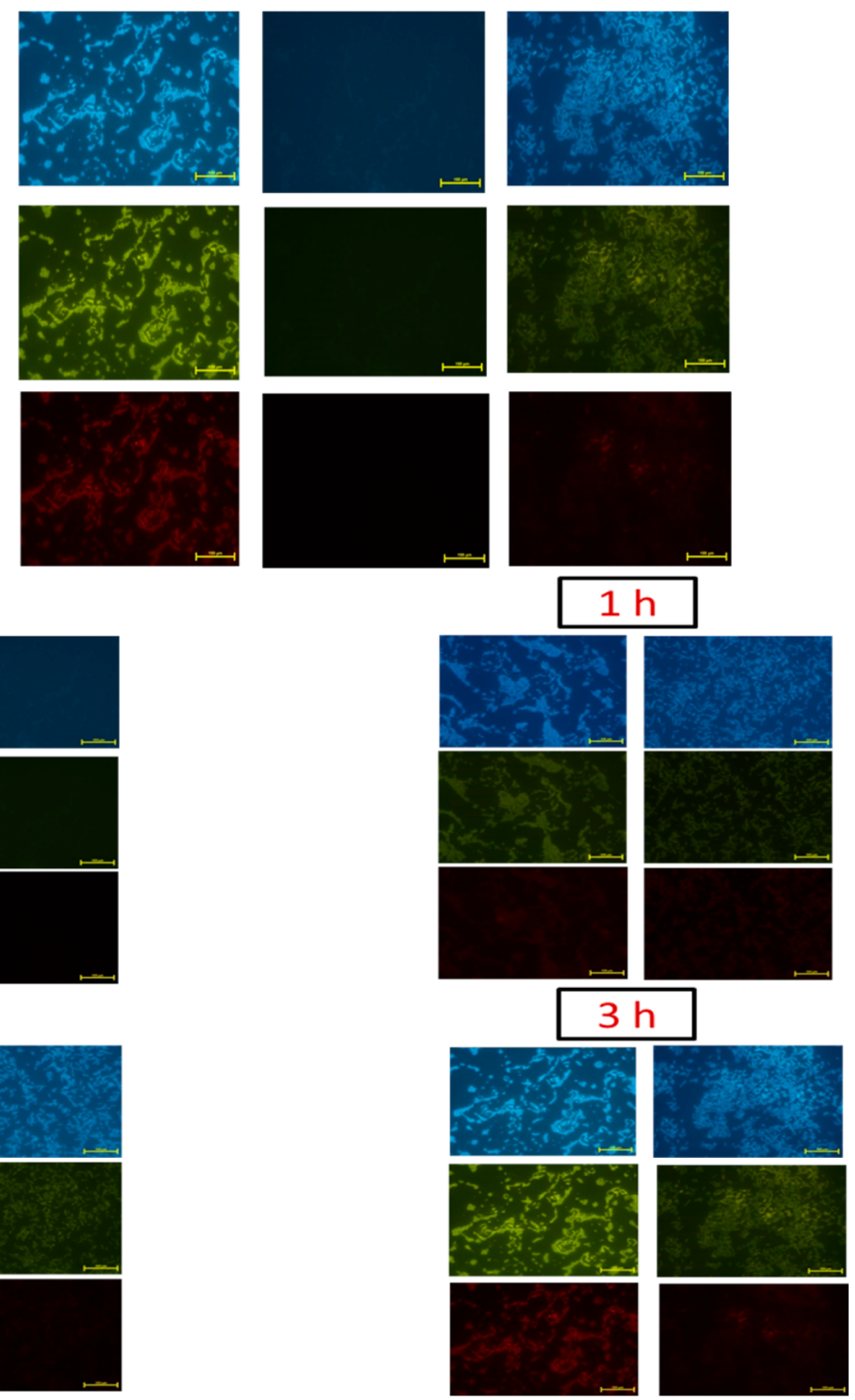

Figure 7. Fluorescence microscopic images of (A) S. aureus and E. coli DH5 $\alpha$ with different treatment of CDP under Blue filter (UV-2A, 330-380 $\mathrm{nm})$, Green filter (B-2A, 450-490 nm), and Red filter (G-2A, 510-560 nm) excitation. (B) Time-dependent fluorescence microscopic images of CPs labeled DH5 $\alpha$ E. coli and S. aureus. Scale bar $100 \mu \mathrm{M}$.

indicates carbonization of curcumin during hydrothermal treatment. Broadening of $3432 \mathrm{~cm}^{-1}$ peaks from curcumin to $\mathrm{CD}$ and $\mathrm{CDP}$ indicates the formation of more $-\mathrm{NH}_{2}$ and $-\mathrm{OH}$ groups. Furthermore, the increase of peak height at $1632 \mathrm{~cm}^{-1}$ signifies the formation of an amide $=\mathrm{C}=\mathrm{O}$ bond according to the used synthesis precursors. ${ }^{3}$

2.5. FE-SEM Analysis (Elemental Mapping and Elemental Composition). Elemental composition was analyzed for CDP by energy-dispersive X-ray spectroscopy (EDS) via FESEM (Figure S3). A significant percentage of oxygen was also found along with the presence of carbon and nitrogen which is in accord with the low positive zeta potential value of CDP in spite of the presence of PEI as a passivating agent. Furthermore, elemental mapping was performed, corroborating the EDS data (Figure S4).
2.6. Ion Sensing Property. The fluorescent-based sensors are an interesting research area being studied around the globe. The metal ion sensing probes are beloved to researchers, among the many. To critically examine the analytical property of synthesized CD from curcumin, with the presence of various metal ions, the fluorescence intensity was observed. The metal ions including $\mathrm{Fe}^{3+}, \mathrm{Ag}^{+}$, and $\mathrm{Cu}^{2+}$ ions caused the reduction in the fluorescent intensity of $\mathrm{CD}$ at the onset of incubation. A change in the fluorescence intensity of $C D\left(0.1 \mathrm{mg} \mathrm{mL}^{-1}\right)$ due to the presence of various metal ions (each at a concentration of $60 \mu \mathrm{M})$ is depicted in Figure 6A.The nonspecific interaction between these metal ions and functional groups of $\mathrm{CD}$ might be the probable reason for the fluorescence quenching. Among them $\mathrm{Fe}^{3+}$ exhibits the strongest quenching efficiency, and this can be exploited for specific $\mathrm{Fe}^{3+}$-based metal sensor application by CD. ${ }^{19,20}$ The concentration dependent fluorescence 
(A)

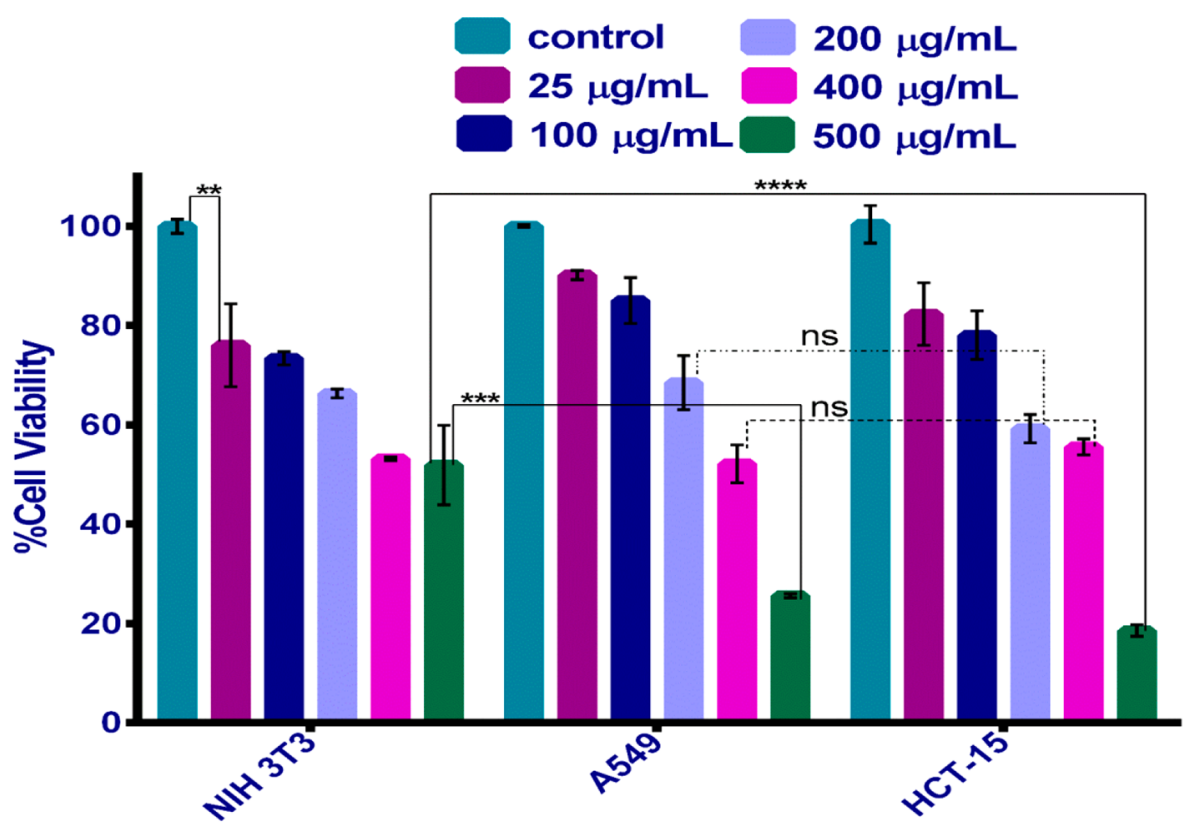

(B)

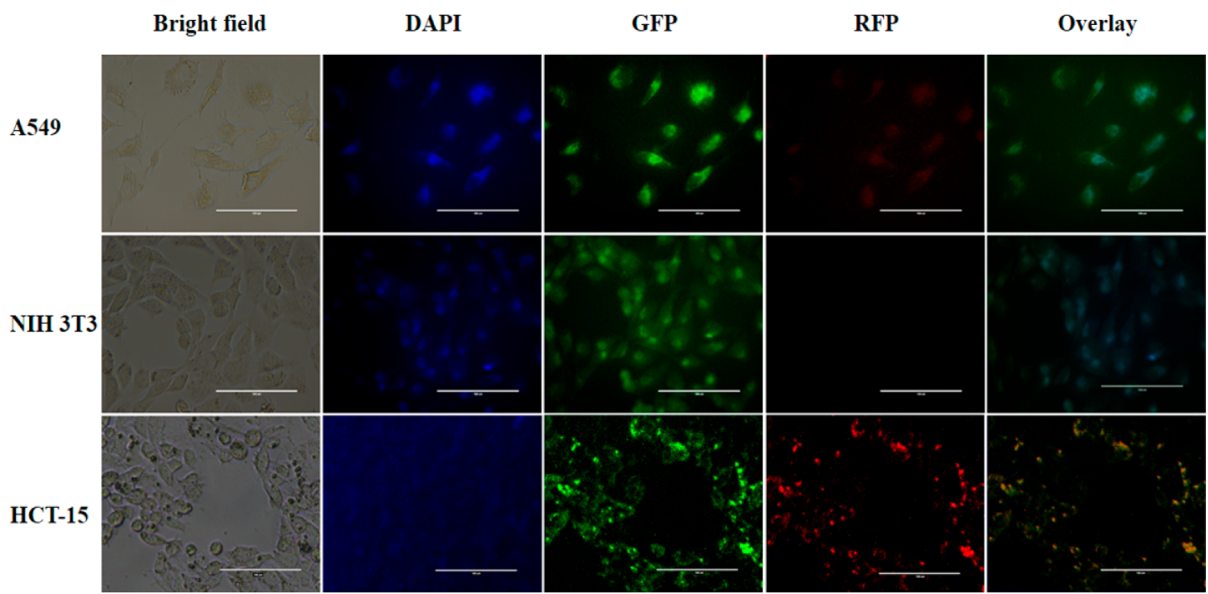

Figure 8. (A) Cell viability analysis of CDP against NIH 3T3, A549, and HCT-15 cells by MTT assay. Two-way ANOVA was carried out by Tukey's multiple comparison test for statistical difference between the group means $(* p<0.05, * * p<0.005, * * *<0.001$, ns-non significant). (B) The multicolor biolabeling potential of CDP in A549, NIH 3T3, and HCT-15 cell lines displays DAPI (Blue), GFP (Green), RFP (Red), and merged images (Overlay). The values are represented as mean \pm SEM $(n=3)$. Scale bar $=100 \mu \mathrm{m}$.

quenching on $\mathrm{Fe}^{3+}$ was further evaluated and obtained with a steady decline in the fluorescence of $\mathrm{CD}$ in a concentrationdependent manner (Figure 6B). Further, the fluorescence quenching efficiency of $\mathrm{CD}$ was described with the SternVolmer plot to extrapolate a correlation coefficient of 0.9928 (a perfect linear correlation) in a concentration range of $0-6 \mu \mathrm{M}$ (Figure $6 \mathrm{C})$. The relative fluorescence response of $\mathrm{CD}\left(F_{0} / F\right)$ was calculated with the following equation

$$
F_{0} / F=K_{\mathrm{sv}}^{*} X+1
$$

where $F_{0}$ and $F$ are the fluorescence intensities of $C D$ in the absence and presence of $\mathrm{Fe}^{3+}$, and $X$ represents the concentration of $\mathrm{Fe}^{3+}$. In the above equation $K_{\mathrm{sv}}$ (slope of the linear fit) was calculated as $11.54 \times 10^{4} \mathrm{~mol}^{-1} \mathrm{dm}^{-3}$. Likewise, the detection limit of $\mathrm{Fe}^{3+}$ was calculated with $3 \sigma / \mathrm{m}$ as $0.62 \mu \mathrm{M}$, where $\sigma$ is the standard deviation of the blank signal $(n=3)$ and $m$ the slope of the linear fit. As per the WHO report $^{21}$ the maximum permissible level of $\mathrm{Fe}^{3+}$ is $5.36 \mu \mathrm{M}$ in drinking water. Since our synthesized $\mathrm{CD}$ can sense the $\mathrm{Fe}^{3+}$ far below the range as mentioned above, it can be a possible $\mathrm{Fe}^{3+}$ sensor. This discrimination outcome for $\mathrm{Fe}^{3+}$ ions resulted from the exceptional coordination between hydroxyl groups of $\mathrm{CD}$ and $\mathrm{Fe}^{3+}$ ions, as compared to existing reports. ${ }^{22,23}$

2.7. Antioxidant Activity of CDP. A few existing reports suggest the antioxidant potential of C-dots. ${ }^{24,25}$ One of the most commonly used ways to conduct the antioxidant activity study is the DPPH-based assay. DPPH is a free radical with nitrogen. It is deep purple in color, upon reaction with an antioxidant, and this deep purple color will eventually change to yellow with respect to the antioxidant potential (Figure S5). Varying concentrations of CDP were mixed with $50 \mu \mathrm{M} \mathrm{DPPH}$ in methanol. After incubation with CDP, we found a decrease in absorbance at $517 \mathrm{~nm}$. As the results were obtained and demonstrated in Figure 6D, the free radical scavenging ability of CDP was found to increase in a concentration-dependent manner. As the initial concentration moved to high concentration (from 5 to $50 \mu \mathrm{g} \mathrm{mL}^{-1}$ ) increment, there was a considerable escalation in the scavenging activity from 22 to $56 \%$. The EC50 value (amount of antioxidant necessary to 

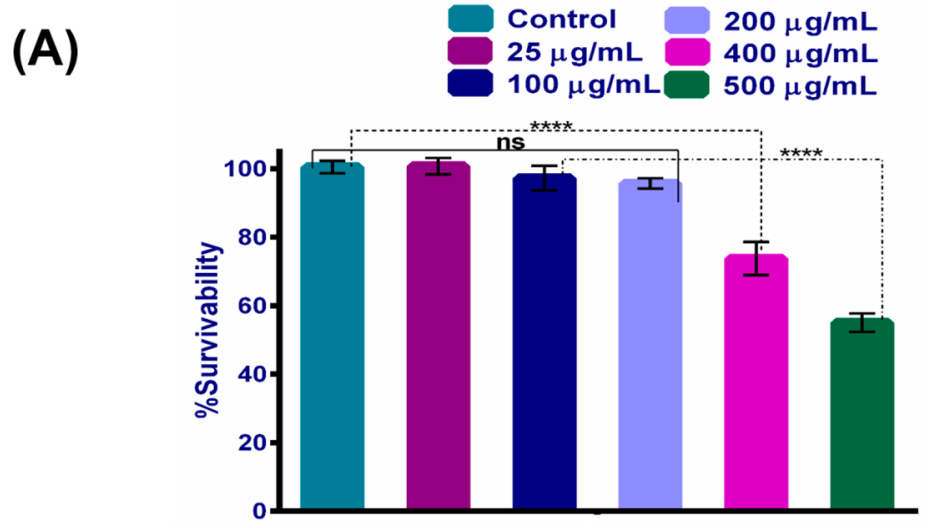

(B)
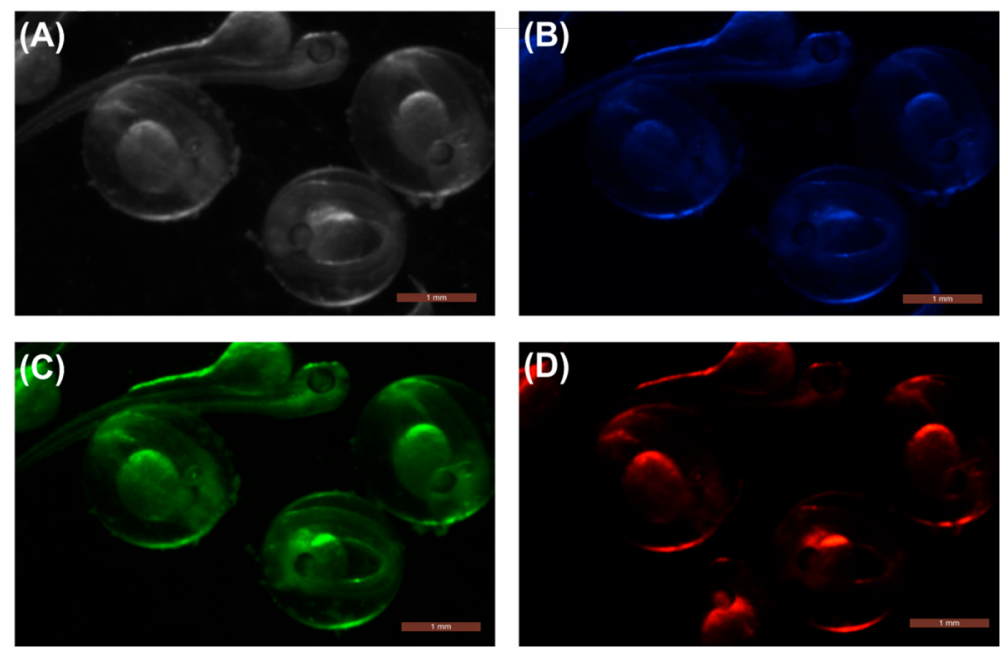

Figure 9. (A) In vivo toxicological assessment with varying concentration of CDP in Zebrafish embryos on a course of 24 h. The values are represented as mean $\pm \operatorname{SEM}(n=3)$. (B) Bright-field (a) and multicolor fluorescence with blue (b), green (c), and red (d) images of zebrafish embryos after soaking for $24 \mathrm{~h}$ in $0.4 \mathrm{mg} / \mathrm{mL}$ concentration of CDP solutions.

decrease the concentration of DPPH by 50\%) of CDP was estimated to be $18.2 \mu \mathrm{g} \mathrm{mL}{ }^{-1}$ from the curve.

2.8. Bacterial Labeling by CDP. Due to the flexibility of CDPs in their multicolor fluorescence, ultrasmall size, better photostability, and high biocompatibility, C-dots can be used as a potential biolabeling agents. On the way to explore its biolabeling activity, E. coli DH5 $\alpha$ and $S$. aureus bacteria were used as Gram-negative and Gram-positive model systems. The bacteria were incubated, and a fluorescence micrograph of labeled bacteria is represented in Figure 7A along with the controls (unlabeled bacteria) for comparison. The CDP labeled bacteria at different excitation resulted in blue, green, and light red color fluorescence. A time-dependent bacterial labeling study was also performed (Figure $7 \mathrm{~B}$ ). The CDP was able to retain their ability to fluoresce the cells after cellular uptake of 3 $\mathrm{h}$ of incubation and displayed excellent multicolor fluorescence. However, no bactericidal effects were observed in bacteria labeling with the CDP at the high concentration of $0.1 \mathrm{mg}$ $\mathrm{mL}^{-1}$. However, the mechanism of bacterial labeling by carbon dots is still unknown. The possible fluorescence labeling might be because the different emissive energy traps ${ }^{26}$ on the surface of the CDP that were produced during hydrothermal treatments enhance the fluorescent emission onset of incubation and excitation.

2.9. MTT Assay. The biomedical imaging application of synthesized CDP was first to critically evaluate for the biocompatibility on both cancer and normal cell lines. In this regard, mouse embryo fibroblast cells (NIH 3T3) were used as a normal cell model, whereas human lung adenocarcinoma cells (A549) and human colorectal adenocarcinoma (HCT-15) were considered as the cancer cell models. The calculated results were plotted on a graph as shown in Figure 8A. The results thus obtained had $\mathrm{IC}_{50}$ values of 580,408 , and $413 \mu \mathrm{g} / \mathrm{mL}$ toward NIH 3T3, A549, and HCT-15 cell lines, respectively. The change in the $\mathrm{IC}_{50}$ values with respect to the cell lines is a clear indication of rapid cellular uptake in the case of cancer cells ${ }^{27}$ correlating its high metabolism and was absent in the case of a normal cell. Upon treatment with CDP, the gradual decrement in the cell viability was shown in each stage and cell lines used. The maximum usable concentration of cell-based bioimaging is found to be $0.4 \mu \mathrm{g} / \mathrm{mL}$ and hence used for multicolor fluorescent imaging. The comparative cytotoxicity was determined by presuming untreated control owing to $100 \%$ viability.

2.10. Bioimaging in Cell Lines. The multicolor biolabeling potential of CDP was explored in A549, NIH 3T3, and HCT-15 cell lines. The bright-field images of CDPtreated cells show intact morphology after the CDP incubation, which shows no detrimental effect of CDP on these cells. The onset of excitation of A549 cells shows excellent blue, green, and less red fluorescence than all other cell lines (Figure 8B). The differentiative staining of the cells was highly visible in A549 cells. This may be because the high metabolism and cellular uptake happen in the cancer cells. ${ }^{26}$ The NIH 3T3 cell 


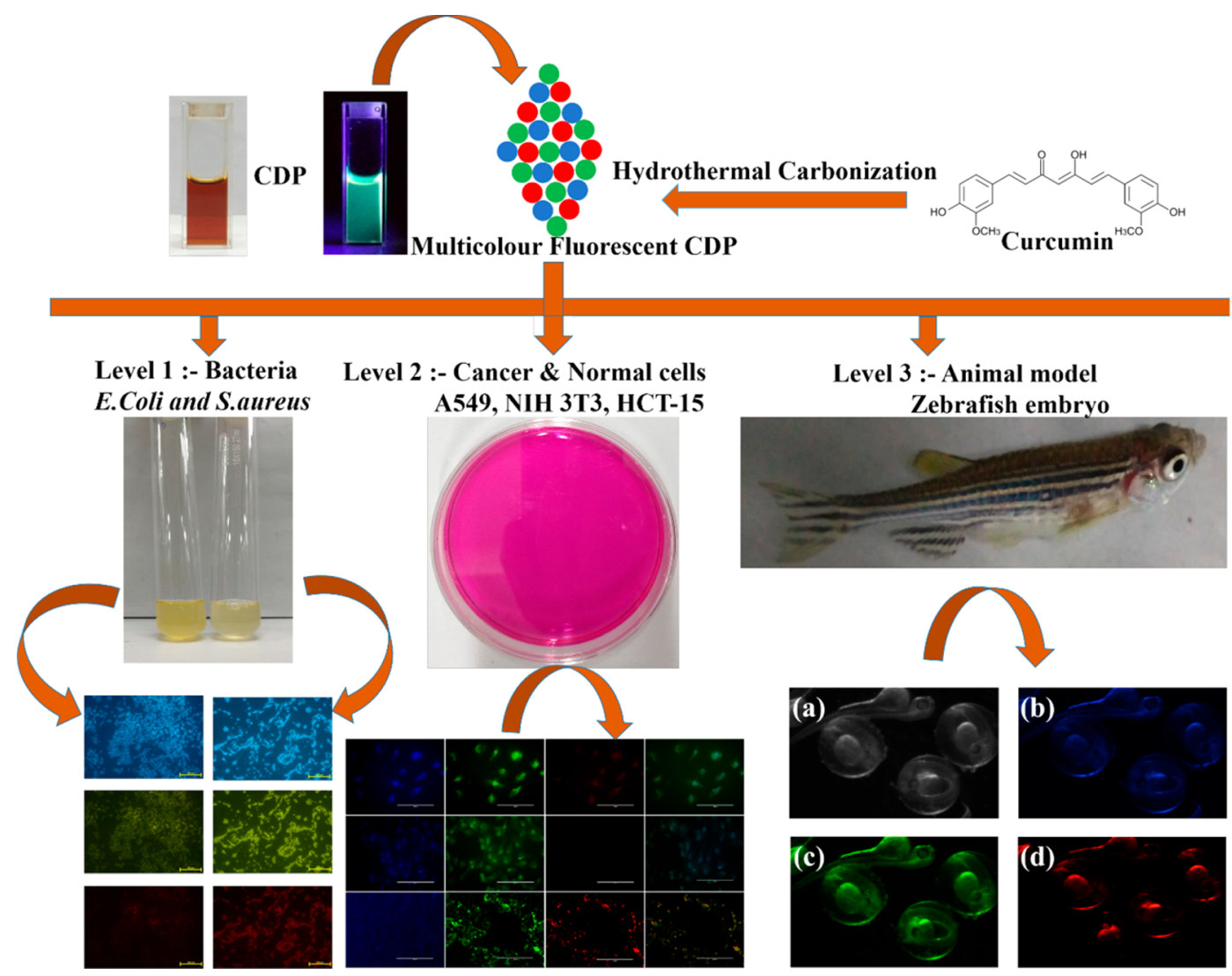

Figure 10. Schematic diagram of multilevel labeling potential of synthesized CDP in prokaryotes to eukaryotes to organism level. Upon cellular uptake and excitation, CDP displays excellent multifluorescent properties.

shows less fluorescent imaging potential when compared with the other two cell lines, and even red fluorescence completely faded. On the other hand, HCT-15 cells show less fluorescent image in blue filter, whereas reasonable images were obtained upon treatment. However, the images showing a ubiquitous distribution of CDP inside the whole cell and little colocalization of CDP in the nucleus were found without cell type discrimination.

2.11. Biocompatibility Studies in Zebrafish. We have conducted zebrafish embryo toxicity (ZET) tests to introduce the embryos of the zebrafish (Danio rerio) to evaluate the toxicity $^{28}$ of CDP on a course of $24 \mathrm{~h}$. After the treatment of the CDP, the uptake of the CDP occurred through the highly permeable chorionic membrane in the 4-HPF embryo. ${ }^{29}$ The toxicity of embryos was get reduced when compared with cell lines, due to the selective nature of the chorionic membrane. The survivability of the embryo shown upon treatment with $\mathrm{CDP}$ at $0.5 \mu \mathrm{g} / \mathrm{mL}$ was about $52 \%$ as shown in Figure 9A. The initial low concentrations have a nonsignificant impact on the zebrafish embryo. The embryos started dying upon treatment after $0.4 \mu \mathrm{g} / \mathrm{mL}$ concentration onward.

2.12. Bioimaging of Zebrafish Embryos. Zebrafish embryos were selected for the organism level of biolabeling due to ease of execution and developmental understanding. As expected from bacterial, cell-based biolabeling, the ubiquitous exhibition of CDP fluorescence was obtained (Figure 9B) simply by soaking $0.4 \mu \mathrm{g} / \mathrm{mL}$ concentration of CDP (arbitrary concentration used after the results obtained from cell-based biolabeling). The multicolor fluorescent microscopic images show the concise uptake of the CDP through the chorionic membrane. Increased fluorescent at the yolk sac region dictates more accumulation in the specified area due to the small size and accessibility of CDP onto the site. ${ }^{30}$ Likewise, from the results obtained from cell-based biolabeling, zebrafish embryos also display higher fluorescence in green then blue and the least in red. The results are obtained as the excellent display of CDP in fluorescent imaging which can be exploited as the best alternative for high-cost contrasting probes.

\section{CONCLUSION}

We have synthesized carbon dots from curcumin as a green source with the aid of hydrothermal reaction in a one-step, facile manner (Figure 10). The nonaqueous soluble curcumin was transformed into a highly hydrophilic carbon dot after hydrothermal synthesis. Furthermore, the surface passivation came into effect with PEI, leading to enhanced bioavailability and fluorescent behaviors. The synthesized carbon dots exhibited excellent fluorescence intensity (in red, green, blue filters), high photostability, and efficient multicolor fluorescent emission upon excitation. The average size of CDP was found to be below $10 \mathrm{~nm}$ with near-spherical shape and was amorphous in nature, which was confirmed with TEM analysis. Also, the potentiality of $\mathrm{CD}$ toward the $\mathrm{Fe}^{3+}$ sensing was taken into consideration and found to be an efficient $\mathrm{Fe}^{3+}$ ion sensor at the micromolar level. The CPDs were also seen to show antioxidant activity. The remarkable multicolor fluorescence emission of CDP services their ability to label bacterial cells with ease. Optimal labeling time was also found by performing time-dependent bacterial labeling. The simple, cost-effective, and efficient CDP was standardized in biolabeling of mammalian cells. The study was further extended to the animal level using zebrafish embryo as a model system. The 
tunable optical behaviors and surface functionalization of CDP depict potential membership in the optical imaging probe category. We, therefore, believe that the curcumin-based green synthesized CDP can effectively overcome the gap between diagnosis and therapy in cancer and infectious diseases.

\section{EXPERIMENTAL SECTION}

4.1. Materials. All the chemicals were of analytical grade and used without any modification. Curcumin, phosphatebuffered saline (PBS), 2,2-diphenyl-1-picrylhydrazyl (DPPH), mercury(II) chloride, lead(II) nitrate, ferrous sulfate heptahydrate, cobalt(II) nitrate hexahydrate, zinc nitrate hexahydrate, ferric chloride anhydrous, and sodium hydroxide $(\mathrm{NaOH})$ were brought from Himedia Laboratories Pvt. Ltd., India, whereas calcium chloride, copper sulfate, and magnesium chloride were provided by SRL (Sisco Research Laboratories) Pvt. Ltd., India. Cadmium acetate and silver nitrate were received from Merck Ltd., India. Solvents like hydrochloric acid $(\mathrm{HCl})$ and $\mathrm{N}$ dimethylformamide (DMF) were from Rankem Pvt. Ltd., India. Nickel(II) acetate tetrahydrate was acquired from SigmaAldrich, USA. Listed solvents such as methanol and dimethyl sulfoxide (DMSO) were received from SD-Fine Chemicals Limited (SDFCL), India. 3-(4,5-Dimethylthiazol-2-yl)-2,5diphenyl-tetrazolium bromide (MTT) was procured from Amresco, USA. Lastly, quinine sulfate was purchased from Loba Chemie Pvt. Ltd., India. Ultrapure water was used to prepare all the solutions.

4.2. Synthesis of Carbon Dots. PEI passivated fluorescent C-dots were synthesized by a one-pot hydrothermal synthesis method as elucidated in Figure 1(A). In a typical synthesis, 0.3 $\mathrm{g}$ of curcumin was added to a solution containing $60 \mathrm{~mL}$ of water and $20 \mathrm{~mL}$ of ethanol. It was followed by addition of 0.4 $\mathrm{g}$ of PEI and stirred vigorously at $80{ }^{\circ} \mathrm{C}$ for $30 \mathrm{~min}$. The color of the solution changed from yellow to red at the onset of PEI addition to curcumin. This solution was later followed by hydrothermal treatment for $12 \mathrm{~h}$ at $200{ }^{\circ} \mathrm{C}$ heating and $15 \mathrm{~kg} /$ $\mathrm{cm}^{2} \mathrm{~g}$ pressure in a nitrogen environment. The dark brown solution was obtained after the system was cooled down and followed with filtering with $125 \mathrm{~mm}$ filter paper and centrifuged at $15000 \mathrm{rpm}$ for $30 \mathrm{~min}$ to separate the less fluorogenic, insoluble deposit from fluorogenic, brown supernatant. The supernatant containing the surface-passivated C-dots was referred to as CDP. Similarly, nonpassivated C-dots were synthesized by a similar scheme but without PEI, and the resulting supernatants were referred to as $\mathrm{CD}$.

4.3. UV-vis Spectroscopy Characterization of C-Dots. The UV-vis double beam spectrophotometer (Lasany, LI2800) was used to find the absorption behavior of the synthesized C-dots. The scanning range for the samples was set at $200-800 \mathrm{~nm}$ with a step size of $0.5 \mathrm{~nm}$. "UV-VIS Analyst" software equipped with the spectrophotometer was used to record and analyze data. All the samples were diluted 100 times for measurements.

4.4. Fluorescence Spectrophotometer. A fluorescence spectrophotometer (Hitachi F-4600, Japan) was used to record the fluorescence spectra of C-dots with a scan speed of 240 $\mathrm{nm} / \mathrm{min}$ with excitation slit width of $5 \mathrm{~nm}$ and emission slit width of $5 \mathrm{~nm}$. The fluorescence behavior was studied by choosing an excitation range of 340-500 nm with $20 \mathrm{~nm}$ increment. 100-fold dilution of the samples was used.

4.5. FTIR Spectrophotometer. An FTIR spectrometer (Thermo Nicolet) was used to study Fourier transform infrared spectra (FTIR) around the range of $4000-400 \mathrm{~cm}^{-1}$ implementing the $\mathrm{KBr}$ pellet method.

4.6. Zeta Potential. Zeta potential measurements were done by a Zetasizer nano-ZS90 series (Malvern Instruments Pvt. Ltd., Germany). A clear disposable zeta cell was used, and $1 \mathrm{~mL}$ of sample was taken in it. Three rounds of measurements each with 15 zeta runs were performed for analysis.

4.7. Field-Emission Scanning Electron Microscope (FESEM). For measurement of elementary composition, $15 \mu \mathrm{L} \mathrm{C}$ dots were dropped on a properly shaped glass slide and then air-dried. This was gold sputtered and was subjected to FESEM provided with EDX (energy-dispersive X-ray detector) working at 15-20 keV. Elemental mapping was also obtained for a different element like carbon, nitrogen, and oxygen.

4.8. Transmission Electron Microscope (TEM). The Cdots were properly diluted with water, and $15 \mu \mathrm{L}$ volume of the same was dropped onto the nonshining side of the TEM grids (carbon-coated copper grid). Images of the sample were taken after proper air drying by TEM (FEI TECHNAI G2) functioning at $120 \mathrm{keV}$. The average particle size was calculated after estimating the size of 40 particles using ImageJ software.

4.9. Photostability Test. Photostability of fluorescent carbon dots was tested by a Hitachi F-4600 fluorescence spectrophotometer equipped with a xenon lamp at an emission wavelength of $446 \mathrm{~nm}$ for CDP and $396 \mathrm{~nm}$ for CD with a scan time of $1800 \mathrm{~s}$. The excitation and emission slit width were kept at $5 \mathrm{~nm}$.

4.10. Quantum Yield Measurements. The quantum yield of the samples was calculated by a single-point scheme by keeping quinine sulfate as a reference which has a quantum yield of about 0.54 at $360 \mathrm{~nm} .^{2}$ The following equation was used to calculate the quantum yield.

$$
Q=Q_{\mathrm{R}} \times \frac{I}{I_{\mathrm{R}}} \times \frac{A_{\mathrm{R}}}{A} \times \frac{\eta^{2}}{\eta_{\mathrm{R}}^{2}}
$$

Here $Q=$ the quantum yield of the sample; $I=$ the integrated emission intensity (area under the emission curve); $\eta=$ refractive index, and $A=$ the optical density.

The subscript $\mathrm{R}$ is the reference fluorophore with a quantum yield which is known. To minimize the reabsorption effects, absorbance in the $10 \mathrm{~mm}$ fluorescence cuvette was kept below 0.1 at the excitation wavelength. The excitation slit width was set to be $1.0 \mathrm{~nm}$, whereas the emission slit width was $2.5 \mathrm{~nm}$. The parameter as mentioned above was set to excite the samples and recorded their fluorescence spectra in the wavelength range of $375-700 \mathrm{~nm}$, respectively.

4.11. Metal Ion Sensing Evaluation. All the stock solutions of metal ion were made from their chemical salts. A final concentration of $60 \mu \mathrm{M}$ of these metal ions was obtained by further diluting with deionized water. CD $(10 \mu \mathrm{L}, 0.1 \mathrm{mg}$ $\mathrm{mL}^{-1}$ ) was added to each of the different metal ion solutions and homogenized well. This was followed by an incubation of $15 \mathrm{~min}$ at ambient temperature provided. An equal volume of all the aliquots was transferred to a 96-well black plate in respective wells, and the fluorescence measurement was performed in the multiplate reader (Biotek, Cytation 3). Similar steps were followed for $\mathrm{Fe}^{3+}$ ion sensing too. For this assay, $10 \mu \mathrm{L}$ of $\mathrm{CD}$ solution was mixed with $1 \mathrm{~mL}$ of $\mathrm{Fe}^{3+}$ salt solutions in varying concentrations. The fluorescence spectrum was documented by setting excitation wavelength at $280 \mathrm{~nm}$.

4.12. Determination of Antioxidant Activity. 2,2Diphenyl-1-picrylhydrazyl (DPPH) free radical assay with few 
modifications was used to measure the antioxidant potential of CDP. Initially, $0.5 \mathrm{~mL}$ of $50 \mathrm{Mm}$ solution of $\mathrm{DPPH}$ was prepared in methanol, and different concentrated samples of CDP were further added. The total volume of all prepared solutions was maintained at $0.6 \mathrm{~mL}$. The samples were incubated for $30 \mathrm{~min}$ along with appropriate controls in the dark environment. An amount of $180 \mu \mathrm{L}$ of the solution was taken in a microcell quartz cuvette, and change of absorbance was noted at $517 \mathrm{~nm}$ in an UV-vis spectrophotometer. The following formula was used to calculate the percentage radical scavenging activity of $\mathrm{CD} .^{31}$

$$
\% \text { scavenging activity }=\left(\mathrm{AD}_{517}-\mathrm{AS}_{517} / \mathrm{AD}_{517}\right) \times 100
$$

where $\mathrm{AD}=\mathrm{DPPH}$ absorbance (without $\mathrm{CDP}$ ) and $\mathrm{AS}=$ the absorbance of the sample solution (with CDP).

4.13. Bacteria Labeling Studies. To study bacteria labeling property, the Gram-positive S. aureus (MTCC 737) and Gram-negative DH5 $\alpha$ E. coli were purchased from IMTECH, India, chosen as model systems. Initially, the overnight grown culture of both bacterial strains was subcultured to obtain a secondary grown culture. From this culture, $1 \mathrm{~mL}$ was taken in an Eppendorf tube and centrifuged for $1 \mathrm{~min}$ at $9000 \mathrm{rpm}$ and then the pellet was washed twice with autoclaved PBS. Finally the obtained pellet was resuspended in $100 \mu \mathrm{L}$ of PBS with $100 \mu \mathrm{L}$ of CDP (0.1 $\mathrm{mg} / \mathrm{mL}$ ) and incubated for $3 \mathrm{~h}$. An amount of $5 \mu \mathrm{L}$ of the samples was deposited on glass slides and allowed to air-dry for fluorescence microscope imaging. Similarly, time-dependent bacterial labeling was performed for optimizing the labeling time.

4.14. Cell Culture. Human lung adenocarcinoma cells (A549), mouse embryo fibroblast cells (NIH 3T3), and human colorectal adenocarcinoma (HCT-15) were procured from the National cell line repository (National Centre for Cell Sciences, Pune, India). Further, A549 and NIH 3T3 cells were grown in Dulbecco's modified Eagle's medium (DMEM), whereas HCT15 grown in Roswell Park Memorial Institute (RPMI-1640) medium was augmented with $10 \% \mathrm{v} / \mathrm{v}$ fetal bovine serum (FBS) and $1 \%$ penicillin-streptomycin solution. The cells were routinely maintained in a $\mathrm{CO}_{2}$ incubator provided with $5 \% \mathrm{CO}_{2}$ and $95 \%$ air at $37{ }^{\circ} \mathrm{C}$ in a humidified chamber.

4.15. MTT Assay. Mitochondrial probe mediated cytotoxicity was assessed by MTT assay. Toxicity of treatment with CDP was colorimetrically estimated by the activity of mitochondrial dehydrogenase enzymes in actively respiring cells to convert the aqueous soluble, yellow MTT into an insoluble, purple formazan as a product. The quantification of purple formazan formation was done at an absorbance of 570 $\mathrm{nm}$ in a microplate reader (Biotek, Cytation 3). Briefly, the cells were seeded in 24-well tissue culture plates at a seeding density of $0.05 \times 10^{6}$ cells per well. Overnight incubation was given for the proper attachment, and later cells underwent incubation with culture media along with varying concentration of CDP in different wells for $24 \mathrm{~h}$. After the incubation, the medium was carefully withdrawn from each well and underwent phosphatebuffered solution (PBS) wash. A fresh medium with $100 \mu \mathrm{L}$ of $5 \mathrm{mg} \mathrm{mL} \mathrm{m}^{-1}$ solution of yellow MTT was poured into each well and kept in the cells for $4 \mathrm{~h}$. The reaction was terminated and followed the addition of $1 \mathrm{~mL}$ of DMSO to solubilize the purple formazan crystals. Finally, the absorbance at $570 \mathrm{~nm}$ of each well was taken for cell viability calculations. The untreated control (with $100 \%$ viability) is taken as the relative value in the viability calculations. The viability [mean $(\%) \pm \operatorname{SEM}, n=3]$ is calculated in the form of an equation as follows:

$$
\begin{gathered}
\text { Cell viability }(\%)=[(\text { A570 }- \text { A690 }) \text { of treated cells } \\
/(\text { A570 - A690 }) \text { of control cells }] \times 100
\end{gathered}
$$

4.16. Bioimaging Studies. The multicolor imaging of live cells with the use of CDP was explored in A549, NIH 3T3, and HCT -15 cell lines. Primarily, the cells were grown at a density of $0.3 \times 10^{6}$ in $6 \mathrm{~cm}$ well culture dishes and grown overnight. This was replaced with a fresh medium mixed with $0.4 \mathrm{mg}$ $\mathrm{mL}^{-1}$ of filter-sterilized CDP $\left(\mathrm{IC}_{50}\right.$ concentration obtained after MTT) in the respective wells and incubated for $24 \mathrm{~h}$. Following PBS wash twice, the CDP fluorescence was bioimaged by an inverted fluorescent microscope (EVOS FL Color, AMEFC 4300) equipped with LED light cubes in respective filters. Bright-field images were taken in transmitted mode, the DAPI filter having an excitation of $360 \mathrm{~nm}$ and emission $447 \mathrm{~nm}$, GFP with excitation of $470 \mathrm{~nm}$ and emission of $525 \mathrm{~nm}$, and RFP with an excitation of $530 \mathrm{~nm}$ and emission of $593 \mathrm{~nm}$ LED light cubes. The overlay images were obtained by superimposing the images taken from all the filters as mentioned above.

4.17. Zebrafish Biocompatibility and Bioimaging. The full genome sequenced wild-type (ASWT) $\operatorname{strain}^{32}$ of zebrafish (Danio rerio) was gifted by Dr. Sridhar Sivasubbu (Developmental Biology and Genomics lab, CSIR-IGIB, India) and was maintained in aquaneering zebrafish facility with continuous flow of 4 step sterilized system water with parameter set as $\mathrm{pH}$ at 7.8 , conductivity of $710 \mu \mathrm{s}, 28{ }^{\circ} \mathrm{C}$ temperature, dissolved oxygen ( $>4 \mathrm{ppm})$, and total gas pressure of $99.9 \%$. The system was provided with an alternative $10 \mathrm{~h}$ dark and $14 \mathrm{~h}$ light cycle. All the zebrafish-based experimental procedures are conducted by following OECD guidelines 203. Sexually matured 6-month aged male and female fish were brought to the breeding tank overnight ( 1 male: 1 female). The next day, fertilized eggs were microscopically selected by their transparent nature. The $4 \mathrm{~h}$ postfertilized (4HPF) embryos were used for further studies. A total of 10 embryos were transferred into a 24-well plate provided with the E3 medium. The concentration-dependent toxicity toward zebrafish embryos was estimated with 10 fish embryos per well upon treatment with CDP to $24 \mathrm{~h}$. Toxicity was measured as follows [mean (\%) \pm SEM, $n=3]$ comparing with untreated control (the no. of samples selected in each case is 10).

$$
\begin{gathered}
\text { \% Survivability }=100-[(\text { No. of live embryos in control } \\
- \text { No. of live embryos in treated well }) \times C]
\end{gathered}
$$

where $C$ is the constant factor with respect to no. of embryos per well to make 100. Therefore,

$$
C=\frac{100 \text { (Percentage Factor })}{10 \text { (No. of embryo in a well })}=10
$$

The CDP-treated zebrafish embryos were bioimaged after a treatment of $24 \mathrm{~h}$. The multicolor fluorescent images were taken in Leica M205 FA attached with a Leica DFC-7000 T camera along with $\mathrm{pE} 300$ Cool LED for the light illumination of fluorescent excitation in DAPI (blue), GFP (green), and RFP (red), whereas bright-field images were taken directly. All images were captured in the Planapo 1.0X objective lens. 


\section{ASSOCIATED CONTENT}

\section{S Supporting Information}

The Supporting Information is available free of charge on the ACS Publications website at DOI: 10.1021/acsomega.7b01323.

Excitation-dependent fluorescence emission spectra (Figure S1), summarized data table for quantum yield calculations (Table S1), selected area electron diffraction (SAED) pattern (Figure S2), energy-dispersive X-ray spectroscopy (EDS) spectra (Figure S3), elemental mapping of CDP (Figure S4), digital photograph of DPPH bleaching with respect to the concentration of CDP in a concentration-dependent manner (Figure S5), and digital photograph of aswt zebrafish embryos with different treatment condition (Figure S6) (PDF)

\section{AUTHOR INFORMATION}

\section{Corresponding Author}

*Tel.: +91-1332-285650. Fax: +91-1332-273560. E-mail: pgopifnt@iitr.ernet.in; genegopi@gmail.com.

\section{ORCID}

Gopinath Packirisamy: 0000-0003-1379-1203

\section{Author Contributions}

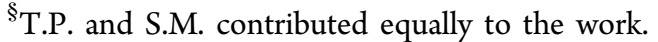

\section{Notes}

The authors declare no competing financial interest.

\section{ACKNOWLEDGMENTS}

This study was partially supported by the Science and Engineering Research Board (no. SR/FT/LS-57/2012) and the Department of Biotechnology (no. BT/PR6804/GBD/27/ $486 / 2012$ ), Government of India. TP is grateful to the Ministry of Human Resource Development (MHRD), Government of India, for the fellowship. SM is thankful to the University Grant Commission (UGC) for providing the fellowship. Sincere thanks to Department of Chemistry and Institute Instrumentation Centre, IIT Roorkee, for the various analytical facilities provided.

\section{REFERENCES}

(1) Gleiter, H. Nanostructured Materials: Basic Concepts and Microstructure. Acta Mater. 2000, 48 (1), 1-29.

(2) Bhushan, B.; Kumar, S. U.; Gopinath, P. Multifunctional Carbon Dots as Efficient Fluorescent Nanotags for Tracking Cells through Successive Generations. J. Mater. Chem. B 2016, 4 (28), 4862-4871.

(3) Sachdev, A.; Matai, I.; Kumar, S. U.; Bhushan, B.; Dubey, P.; Gopinath, P. A Novel One-Step Synthesis of PEG Passivated Multicolour Fluorescent Carbon Dots for Potential Biolabeling Application. RSC Adv. 2013, 3 (38), 16958-16961.

(4) Matai, I.; Sachdev, A.; Gopinath, P. Self-Assembled Hybrids of Fluorescent Carbon Dots and PAMAM Dendrimers for Epirubicin Delivery and Intracellular Imaging. ACS Appl. Mater. Interfaces 2015, 7 (21), 11423-11435.

(5) Yang, X.; Peng, L.; Zong, J.; Zhu, Y. Preparation of Photoluminescent Carbon Dots-Embedded Polyelectrolyte Microcapsules. Particuology 2013, 11 (3), 334-339.

(6) Li, H.; Liu, R.; Liu, Y.; Huang, H.; Yu, H.; Ming, H.; Lian, S.; Lee, S.-T.; Kang, Z. Carbon Quantum dots/Cu2O Composites with Protruding Nanostructures and Their Highly Efficient (near) Infrared Photocatalytic Behavior. J. Mater. Chem. 2012, 22 (34), 17470-17475.

(7) Singh, D. K.; Manam, J. Investigation of Structural, Spectral and Photometric Properties of CaTiO3:Dy3+ Nanophosphors for the Lighting Applications. Electron. Mater. Lett. 2017, 13, 292-301.
(8) Zhang, H.; Ming, H.; Lian, S.; Huang, H.; Li, H.; Zhang, L.; Liu, Y.; Kang, Z.; Lee, S.-T. Fe2O3/carbon Quantum Dots Complex Photocatalysts and Their Enhanced Photocatalytic Activity under Visible Light. Dalt. Trans. 2011, 40 (41), 10822-10825.

(9) Li, H.; Zhu, Y.; Cao, H.; Yang, X.; Li, C. Preparation and Characterization of Photocatalytic Carbon Dots-Sensitized Electrospun Titania Nanostructured Fibers. Mater. Res. Bull. 2013, 48 (2), 232-237.

(10) Wang, R.; Li, G.; Dong, Y.; Chi, Y.; Chen, G. Carbon Quantum Dot-Functionalized Aerogels for NO2 Gas Sensing. Anal. Chem. 2013, 85 (17), 8065-8069.

(11) Hu, S.-L.; Niu, K.-Y.; Sun, J.; Yang, J.; Zhao, N.-Q.; Du, X.-W. One-Step Synthesis of Fluorescent Carbon Nanoparticles by Laser Irradiation. J. Mater. Chem. 2009, 19 (4), 484-488.

(12) Zhang, M.; Bai, L.; Shang, W.; Xie, W.; Ma, H.; Fu, Y.; Fang, D.; Sun, H.; Fan, L.; Han, M.; Liu, C.; Yang, S. Facile Synthesis of WaterSoluble, Highly Fluorescent Graphene Quantum Dots as a Robust Biological Label for Stem Cells. J. Mater. Chem. 2012, 22 (15), 74617467.

(13) Yang, Y.; Cui, J.; Zheng, M.; Hu, C.; Tan, S.; Xiao, Y.; Yang, Q.; Liu, Y. One-Step Synthesis of Amino-Functionalized Fluorescent Carbon Nanoparticles by Hydrothermal Carbonization of Chitosan. Chem. Commun. 2012, 48 (3), 380-382.

(14) Zhu, C.; Zhai, J.; Dong, S. Bifunctional Fluorescent Carbon Nanodots: Green Synthesis via Soy Milk and Application as MetalFree Electrocatalysts for Oxygen Reduction. Chem. Commun. 2012, 48 (75), 9367-9369.

(15) Yang, C.; Thomsen, R. P.; Ogaki, R.; Kjems, J.; Teo, B. M. Ultrastable Green Fluorescence Carbon Dots with a High Quantum Yield for Bioimaging and Use as Theranostic Carriers. J. Mater. Chem. B 2015, 3 (22), 4577-4584.

(16) Liu, M.; Ji, J.; Zhang, X.; Zhang, X.; Yang, B.; Deng, F.; Li, Z.; Wang, K.; Yang, Y.; Wei, Y. Self-Polymerization of Dopamine and Polyethyleneimine: Novel Fluorescent Organic Nanoprobes for Biological Imaging Applications. J. Mater. Chem. B 2015, 3 (17), 3476-3482.

(17) Gogoi, B.; Sarma, N. S. Enhanced Fluorescence Quenching of Hemin Detected by a Novel Polymer of Curcumin. RSC Adv. 2013, 3 (21), 7747-7750.

(18) Park, Y.; Yoo, J.; Lim, B.; Kwon, W.; Rhee, S.-W. Improving the Functionality of Carbon Nanodots: Doping and Surface Functionalization. J. Mater. Chem. A 2016, 4 (30), 11582-11603.

(19) En, D.; Guo, Y.; Chen, B.-T.; Dong, B.; Peng, M.-J. CoumarinDerived $\mathrm{Fe} 3+-S e l e c t i v e$ Fluorescent Turn-off Chemosensors: Synthesis, Properties, and Applications in Living Cells. RSC Adv. 2014, 4 (1), 248-253.

(20) Bao, X.; Shi, J.; Nie, X.; Zhou, B.; Wang, X.; Zhang, L.; Liao, H.; Pang, T. A New Rhodamine B-Based "on-off" Chemical Sensor with High Selectivity and Sensitivity toward Fe3+ and Its Imaging in Living Cells. Bioorg. Med. Chem. 2014, 22 (17), 4826-4835.

(21) Gorchev, H. G.; Ozolins, G. WHO Guidelines for DrinkingWater Quality. WHO Chron. 2011, 38 (3), 104-108.

(22) Gong, X.; Lu, W.; Paau, M. C.; Hu, Q.; Wu, X.; Shuang, S.; Dong, C.; Choi, M. M. F. Facile Synthesis of Nitrogen-Doped Carbon Dots for Fe3+ Sensing and Cellular Imaging. Anal. Chim. Acta 2015, 861, 74-84.

(23) Teng, X.; Ma, C.; Ge, C.; Yan, M.; Yang, J.; Zhang, Y.; Morais, P. C.; Bi, H. Green Synthesis of Nitrogen-Doped Carbon Dots from Konjac Flour With "off-On" fluorescence by $\mathrm{Fe} 3+$ and L-Lysine for Bioimaging. J. Mater. Chem. B 2014, 2 (29), 4631-4639.

(24) Das, B.; Dadhich, P.; Pal, P.; Srivas, P. K.; Bankoti, K.; Dhara, S. Carbon Nanodots from Date Molasses: New Nanolights for the in Vitro Scavenging of Reactive Oxygen Species. J. Mater. Chem. B 2014, 2 (39), 6839-6847.

(25) Das Purkayastha, M.; Manhar, A. K.; Das, V. K.; Borah, A.; Mandal, M.; Thakur, A. J.; Mahanta, C. L. Antioxidative, Hemocompatible, Fluorescent Carbon Nanodots from an "End-ofPipe" Agricultural Waste: Exploring Its New Horizon in the FoodPackaging Domain. J. Agric. Food Chem. 2014, 62 (20), 4509-4520. 
(26) Lim, S. Y.; Shen, W.; Gao, Z. Carbon Quantum Dots and Their Applications. Chem. Soc. Rev. 2015, 44 (1), 362-381.

(27) Cairns, R. A.; Harris, I. S.; Mak, T. W. Regulation of Cancer Cell Metabolism. Nat. Rev. Cancer 2011, 11 (2), 85-95.

(28) Klüver, N.; König, M.; Ortmann, J.; Massei, R.; Paschke, A.; Kühne, R.; Scholz, S. Fish Embryo Toxicity Test: Identification of Compounds with Weak Toxicity and Analysis of Behavioral Effects To Improve Prediction of Acute Toxicity for Neurotoxic Compounds. Environ. Sci. Technol. 2015, 49 (11), 7002-7011.

(29) Adams, S. L.; Zhang, T.; Rawson, D. M. The Effect of External Medium Composition on Membrane Water Permeability of Zebrafish (Danio Rerio) Embryos. Theriogenology 2005, 64 (7), 1591-1602.

(30) Kang, Y.-F.; Li, Y.-H.; Fang, Y.-W.; Xu, Y.; Wei, X.-M.; Yin, X.-B. Carbon Quantum Dots for Zebrafish Fluorescence Imaging. Sci. Rep. 2015, 5, 11835.

(31) Sachdev, A.; Gopinath, P. Green Synthesis of Multifunctional Carbon Dots from Coriander Leaves and Their Potential Application as Antioxidants, Sensors and Bioimaging Agents. Analyst 2015, 140 (12), 4260-4269.

(32) Singh, A. R.; Sivadas, A.; Sabharwal, A.; Vellarikal, S. K.; Jayarajan, R.; Verma, A.; Kapoor, S.; Joshi, A.; Scaria, V.; Sivasubbu, S. Chamber Specific Gene Expression Landscape of the Zebrafish Heart. PLoS One 2016, 11 (1), e0147823. 